\title{
ACCP IN POLYNOMIAL RINGS: A COUNTEREXAMPLE
}

\author{
WILLIAM J. HEINZER AND DAVID C. LANTZ
}

(Communicated by Wolmer V. Vasconcelos)

\begin{abstract}
We describe an example showing that ACCP need not extend from a ring to a polynomial ring over it.
\end{abstract}

A ring $R$ (always commutative with unity) is said to satisfy the ascending chain condition on principal ideals (ACCP) if, for any infinite ascending chain of principal ideals $a_{1} R \subseteq a_{2} R \subseteq \cdots$, there is a positive integer $n$ for which $a_{n} R=a_{n+1} R=\cdots \quad$ (cf. [Gi]). This property has been studied, even in the case of a noncommutative ring, in a number of papers, for example, [AAZ, $\mathrm{AN}, \mathrm{Gr}, \mathrm{N}, \mathrm{R}]$. It is well known and easy to see that if $R$ is an integral domain satisfying ACCP, then for any family $X$ of indeterminates, the polynomial ring $R[X]$ also satisfies ACCP. (In [Gr], where it seems to be asserted that this holds for any ring, there appears to be a tacit hypothesis of domain.)

Example. A ring $R$ that satisfies ACCP but for which the polynomial ring $R[x]$, in a single indeterminate $x$, does not satisfy ACCP. Let $k$ be a field and $A_{1}, A_{2}, \ldots$ be indeterminates over $k$, and set

$$
S=k\left[A_{1}, A_{2}, \ldots\right] /\left(\left\{A_{n}\left(A_{n-1}-A_{n}\right): n \geq 2\right\}\right) k\left[A_{1}, A_{2}, \ldots\right] .
$$

Denote by $a_{n}$ the image of $A_{n}$ in $S$ and by $R$ the localization of $S$ at the ideal $\left(a_{1}, a_{2}, \ldots\right) S$. We note two facts about these rings: (1) the elements of $S$ that become units in $R$ are nonzerodivisors, so $R$ contains (an isomorphic copy of) $S$; and (2) in $S$, no power of $a_{n-1}$ annihilates the difference $a_{n-1}-a_{n}$. For (1), note that, as the factor ring of a polynomial ring over $k$ by a homogeneous ideal (in total degree in the $A_{n}$ 's), $S$ is a graded ring. Thus, we can refer to the order of an element of $S$, i.e., the degree of the smallest-degree nonzero term in that element; and for elements $f, g$ in $S$, ord $(f g) \geq \operatorname{ord}(f)+\operatorname{ord}(g)$. Since an element outside $\left(a_{1}, a_{2}, \ldots\right) S$ has unit degree-0 term, its product with any

Received by the editors July 13, 1993.

1991 Mathematics Subject Classification. Primary 13E99, 13B25, 13 A02.

The first author gratefully acknowledges support under NSF Grant DMS 91-01176. The second author gratefully acknowledges the hospitality of Purdue University while this work was done. The authors are grateful to Ralf Schmücker for re-awakening interest in the properties of ACCP and $n$-acc.

The second author will present this example to the conference on commutative algebra in connection with the 1994 John H. Barrett Memorial Lectures, University of Tennessee-Knoxville, April 7-9, 1994. 
nonzero element $g$ of $S$ has the same order as $g$; in particular, the product is not zero.

For (2), we regard $S$ as the limit of the rings $S_{n}$ where $S_{1}=k\left[a_{1}\right]$ and

$$
S_{n}=S_{n-1}\left[a_{n}\right]=S_{n-1}\left[A_{n}\right] / A_{n}\left(a_{n-1}-A_{n}\right) S_{n-1}\left[A_{n}\right]
$$

for $n \geq 2$. Since $A_{n}\left(a_{n-1}-A_{n}\right)$ is the negative of a monic polynomial of degree $2, S_{n}$ is a free module over $S_{n-1}$ on the generators $1, a_{n}$. Thus, $S_{n-1}$ is a subring of $S_{n}$, and a power of $a_{n-1}$ annihilates $a_{n-1}-a_{n}$ only if $a_{n-1}$ is nilpotent. Assume $a_{n-1}^{m}=0$ where, without loss of generality, $m>n-2$. Then, using the defining relations of $S$, we have $a_{n-1}^{m}=a_{1}^{m-n+2} a_{2} a_{3} \cdots a_{n-1}$. Since each $a_{k}$ is an element of a free basis over $S_{k-1}$, we conclude that $a_{1}$ is nilpotent. But that is a contradiction, since $S_{1}$ is (isomorphic to) the polynomial ring in the indeterminate $a_{1}$ over $k$.

To see that $R$ satisfies ACCP, we again use the grading on $S$ : Suppose we have $f_{1} R \subset f_{2} R$, where $f_{1}, f_{2}$ are chosen from $S$. Then since elements of $S$ of order 0 are units in $R$, we must have elements $g, h$ in $S$ for which $\operatorname{ord}(g)>0, \operatorname{ord}(h)=0$, and $f_{2}(g / h)=f_{1}$ in $R$, so that in $S$, using (1) above, we have $f_{1} h=f_{2} g$. It follows that $\operatorname{ord}\left(f_{1}\right)>\operatorname{ord}\left(f_{2}\right)$. Since orders in $S$ are bounded below by 0 , it follows that $R$ satisfies ACCP.

Now in $R[x]$, we have

$$
\left(a_{n} x+1\right)\left(\left(a_{n-1}-a_{n}\right) x+1\right)=a_{n-1} x+1
$$

for each $n \geq 2$, so

$$
\left(a_{1} x+1\right) R[x] \subseteq\left(a_{2} x+1\right) R[x] \subseteq \cdots .
$$

To see that these containments are proper, suppose by way of contradiction that, for some $n \geq 2,\left(a_{n-1} x+1\right) b(x)=a_{n} x+1$ where $b(x)$ is the polynomial $b(x)=b_{0}+b_{1} x+\cdots$. Then we must have $b_{0}=1$ and by induction $b_{m}=$ $(-1)^{m-1} a_{n-1}^{m-1}\left(a_{n}-a_{n-1}\right)$ for each $m>0$. By (2), all the $b_{m}$ are nonzero, so $b(x)$ is not a polynomial, which is the desired contradiction.

Remark. It is shown in [HL, (3.8) and (3.9)] that if $R$ is a quasilocal ring having the property that the annihilator of each finitely generated ideal in $R$ has only finitely many minimal primes or if $R$ is of dimension zero, then ACCP extends to a polynomial ring over $R$. The $R$ in the example above is quasilocal of dimension one and has a countably infinite number of minimal primes. Moreover, by using [HL, Proposition 2.1] it follows that $R$ satisfies the ascending chain condition on $n$-generated ideals for every positive integer $n$ (i.e., $R$ has "pan-acc").

\section{REFERENCES}

[AAZ] D. D. Anderson, D. F. Anderson, and M. Zafrullah, Factorization in integral domains, J. Pure Appl. Algebra 69 (1990), 1-19.

[AN] M. E. Antunes-Simões and A. M. Nicolas, Anneaux n-acc, Comm. Algebra 12 (1984), 1653-1665.

[Gi] R. Gilmer, Multiplicative ideal theory, Marcel Dekker, New York, 1972.

[Gr] A. Grams, Atomic rings and the ascending chain condition for principal ideals, Proc. Cambridge Philos. Soc. 75 (1974), 321-329. 
[HL] W. Heinzer and D. Lantz, Commutative rings with ACC on n-generated ideals, J. Algebra 80 (1983), 261-278.

[N] A. M. Nicolas, Thèse - modules factorables, modules n-acc, Université de Limoges, 1981.

[R] G. Renault, Sur des conditions de chaînes ascendantes dans des modules libres, J. Algebra 47 (1977), 268-275.

Department of Mathematics, Purdue University, West Lafayette, Indiana 47907-1395

E-mail address: heinzer@math.purdue.edu

Department of Mathematics, Colgate University, 13 Oak Drive, Hamilton, New York 13346-1398

E-mail address: dlantz@center.colgate.edu 